\title{
Late to early velocity index as a predictor of iatrogenic femoral artery pseudoaneurysm recurrence in patients treated with ultrasound-guided thrombin injection
}

\author{
Jacek Kurzawski ${ }^{1}$, Agnieszka Janion-Sadowska ${ }^{1}$, Łukasz Zandecki ${ }^{2}$, Marianna Janion², Marcin Sadowski
}

${ }^{1}$ Department of Cardiology, Świętokrzyskie Cardiology Centre, Kielce, Poland ${ }_{2}^{2}$ Institute of Medical Sciences, Jan Kochanowski University, Kielce, Poland

Submitted: 29 November 2018; Accepted: 3 April 2019;

Online publication: 21 May 2019

Arch Med Sci 2021; 17 (3): 652-661

DOI: https://doi.org/10.5114/aoms.2019.85249

Copyright $\odot 2019$ Termedia \& Banach

\section{Abstract}

Introduction: Ultrasound-guided thrombin injection (UGTI) is the preferred treatment of pseudoaneurysms (psA). The potential risk of complications increases with the number of UGTI treatments needed for complete psA obliteration. Identification of risk factors for recurrent psA is needed.

Material and methods: In total, 508 patients with femoral artery psA underwent UGTI, followed by ultrasound examination repeated twice, at 1-week intervals, to assess UGTI effectiveness. In cases of psA recurrence, the procedure was repeated. Clinical and ultrasound data were prospectively collected.

Results: The psA recurrence occurred in $76(15 \%)$ patients. UGTI was repeated twice in $49(64 \%)$, three times in $15(20 \%)$ and more than three times in $12(16 \%)$ patients. The median thrombin dose was 150 IU (80-250 IU), and was lower in initial procedures than repeated UGTI $(p=0.025)$. The median psA volume was $2.26 \mathrm{ml}(0.86-5.47 \mathrm{ml})$. The median length of the communicating channel was $4 \mathrm{~mm}(0-12 \mathrm{~mm})$. A time interval between vessel catheterization and UGTI greater than 7 days $(p<0.001)$, a late to early velocity index (LEVI) of $<0.2$ identified during the outflow phase $(p<0.001)$, a psA volume $>5 \mathrm{ml}(p=0.032)$, and a short communicating channel between the psA and the artery $(p=0.037)$ predicted psA recurrence. Antiplatelet and anticoagulant agents did not increase the risk.

Conclusions: The LEVI and time interval between artery cannulation and UGTI treatment are strong parameters identifying patients at risk of psA recurrence. The psA volume and communicating channel length are less substantial risks, but still significant. Concomitant antiplatelet and anticoagulant therapy do not affect the success rate of UGTI.

Key words: pseudoaneurysm, recanalization, thrombin injection.

\section{Introduction}

A pseudoaneurysm (psA) develops as a consequence of arterial wall rupture or injury. It complicates up to $8 \%$ of interventional cardiovascular procedures [1-4]. Ultrasound-guided thrombin injection (UGTI) is currently the first-line treatment for patients with psA, with a success rate exceeding 94\% [1, 3-12]. The remaining patients are referred for surgical repair or stent graft implantation, or are rarely treated with coil embolization or various other substances [2, 4, 13-20]. The main objective of

\author{
Corresponding author: \\ Assoc. Prof. Marcin Sadowski \\ $\mathrm{MD}, \mathrm{PhD}$ \\ Institute of Medical Sciences \\ Faculty of Medicine \\ and Health Sciences \\ Jan Kochanowski University \\ Al. IX Wieków Kielc 19 A \\ 25-317 Kielce, Poland \\ Phone: +48 606906454 \\ E-mail: emsad@o2.pl
}


UGTI is to promote thrombus formation within the psA lumen, ceasing blood leakage from the arterial lumen, and thus the occlusion (embolization) of the psA. It is estimated that recurrence may follow obliteration using UGTI in $2.5 \%$ to $13 \%$ of psA patients. As a result, in cases where a repeat procedure is warranted, complications can occur which are due to prolonged hospitalization and thrombin treatment, which is a very potent pharmacological agent $[3,4,10,11]$. Moreover, there still exists some ambiguity on the recommended dosage of thrombin, and its therapeutic range. It is also unknown which patients are most at risk for psA recurrence. Significant results from the literature are summarized in Table I. The objective of the present study was to define predictors of psA recurrence after UGTI treatment.

\section{Material and methods}

From June 2004 to January 2017 a total of 46,181 patients with transcatheter cardiovascular interventions (i.e. coronary angiography and angioplasty, electrophysiological examination and ablation, peripheral angiography, intra-aortic balloon pump insertion) underwent a prospective ul- trasound screening for the presence of transfemoral access site-related complications. In 515 (1.1\%) cases femoral artery psA was confirmed. Of these, $508(98.6 \%)$ underwent UGTI, $3(0.6 \%)$ were referred to vascular surgery, $2(0.4 \%)$ had a spontaneous psA thrombosis, and in $1(0.2 \%)$ patient an effective ultrasound-guided compression was performed. psAs were detected using the following devices: Hewlett-Packard Sonos 2000, Sequoia C256, Sequoia C512 and General Electric Vivid E9 with linear 7-10 MHz and sector 2.5-3.5 MHz probes. Data were acquired using two-dimensional imaging and color-Doppler mapping. Blood flow across the psA neck was assessed using pulsewave and continuous-wave spectral Doppler. A definitive diagnosis was made after visualization of the neck and/or communicating channel, as well as a cavity or cavities with at least one current inflow (Figures $1 \mathrm{~A}, \mathrm{~B}$ ). The following qualitative and quantitative parameters (considered as independent variables) were analyzed: age, sex, type of cannulated vessel (i.e. artery or vein) and the exact cannula location (i.e. common, superficial or deep femoral artery), sheath size, hemostatic agents, inpatient versus outpatient diagnostic setting,

Table I. Significant papers on femoral artery pseudoaneurysm recurrence after successful obliteration following ultrasound-guided thrombin injection

\begin{tabular}{|lccccc|}
\hline Cited paper & No. of cases & $\begin{array}{c}\text { Thrombin dose* } \\
{[\text { IU] }}\end{array}$ & $\begin{array}{c}\text { Success rate } \\
(\%)^{\star *}\end{array}$ & $\begin{array}{c}\text { Recurrence } \\
\text { rate, } n(\%)\end{array}$ & $\begin{array}{c}\text { Complication } \\
\text { rate, } n(\%)\end{array}$ \\
\hline Paulson, 2001 [9] & 114 & 306 & 90 & $11(9.6)$ & $4(4)$ \\
\hline Khoury, 2002 [12] & 131 & NA & 96 & $9(6.9)$ & $3(3)$ \\
\hline Maleux, 2003 [1] & 101 & 200 & 98 & $5(4.9)$ & 0 \\
\hline Krueger, 2005 [3] & 240 & 425 & 94 & $14(5.8)$ & $2(0.8)$ \\
\hline Hofmann, 2007 [4] & 595 & 400 & 89 & $38(6.4)$ & $4(1.0)$ \\
\hline Schneider, 2009 [10] & 274 & 450 & 97 & $7(2.5)$ & $1(0.4)$ \\
\hline Kurzawski, 2016 [11] & 353 & 235 & 98 & $46(13.0)$ & $1(0.28)^{* * *}$ \\
\hline
\end{tabular}

${ }^{\star}$ Mean thrombin dose per patient, ${ }^{\star *}$ of the first procedure, ${ }^{\star * \star} K u$ rzawski et al. reported one severe complication (pulmonary embolism) and $54(15.6 \%)$ mild complications related to distal embolization without clinical sequelae. IU - international units.
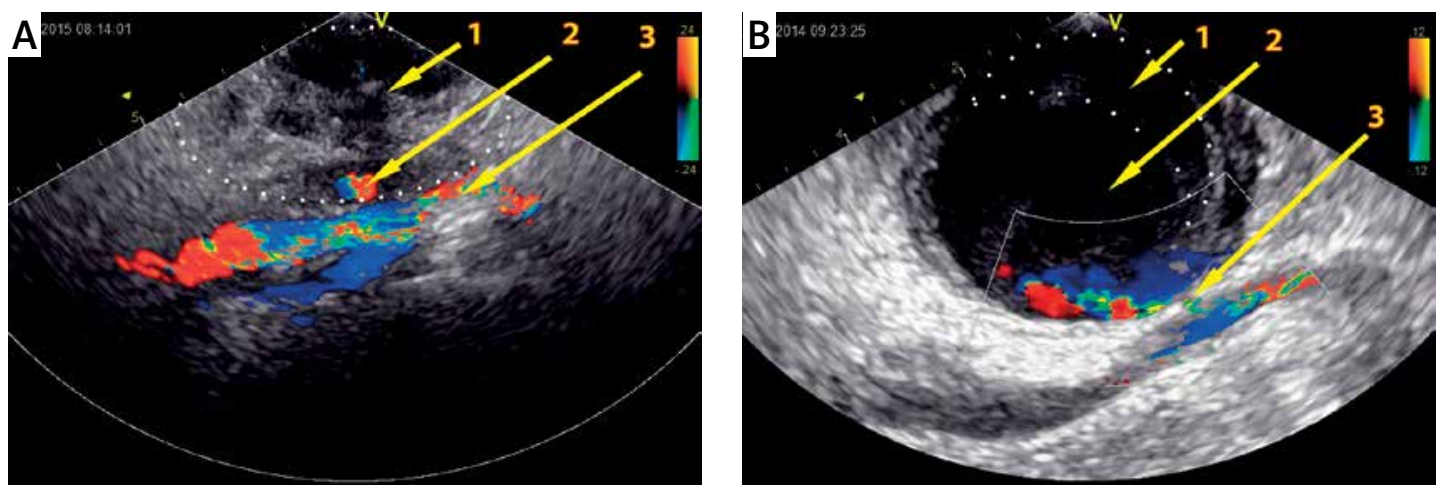

Figure 1. Ultrasound images with color flow mapping of the inguinal area with two different femoral artery pseudoaneurysms. A - Large thrombus (1) and small pseudoaneurysm chamber (2), common femoral artery (3). B - large mural thrombus (1), large pseudoaneurysm chamber (2) and channel (3) between aneurysm and artery 
the time interval of vessel puncture to UGTI treatment, the number and volume of psA cavities, the length of the communicating channel, the number of UGTI attempts, the thrombin dose given during the first UGTI range (i.e. < $200 \mathrm{IU}, 200-600 \mathrm{IU}$, $>600 \mathrm{IU})$, as well as total thrombin doses per patient. Theoretical psA volume was obtained by using the volume of an ellipsoid formula $4 / 3 \pi(a \times b \times$ $c)$; where $a, b$ and $c$ are radii along each axis. A repeat ultrasonography was performed 1-3 days after the initial test and at 7 days following UGTI. UGTI-related complications were assessed as previously described [11]. The effectiveness of UGTI treatment was confirmed by a thrombus in the entire psA chamber, visible also with no inflow towards the psA (either in the communicating channel or in the chamber itself), which was detected by color or pulsed-wave Doppler. The outflow Doppler spectrum during diastole, between two consecutive systolic inflow phases, together with late to early velocity index (LEVI, a newly proposed parameter) in the outflow spectrum, was also analyzed (Figure 2). The primary endpoint was psA recurrence following the initial UGTI and was defined as any inflow toward the psA (either in the communicating channel or in the chamber itself) detectable by color or pulsed-wave Doppler. The study population was subdivided into two
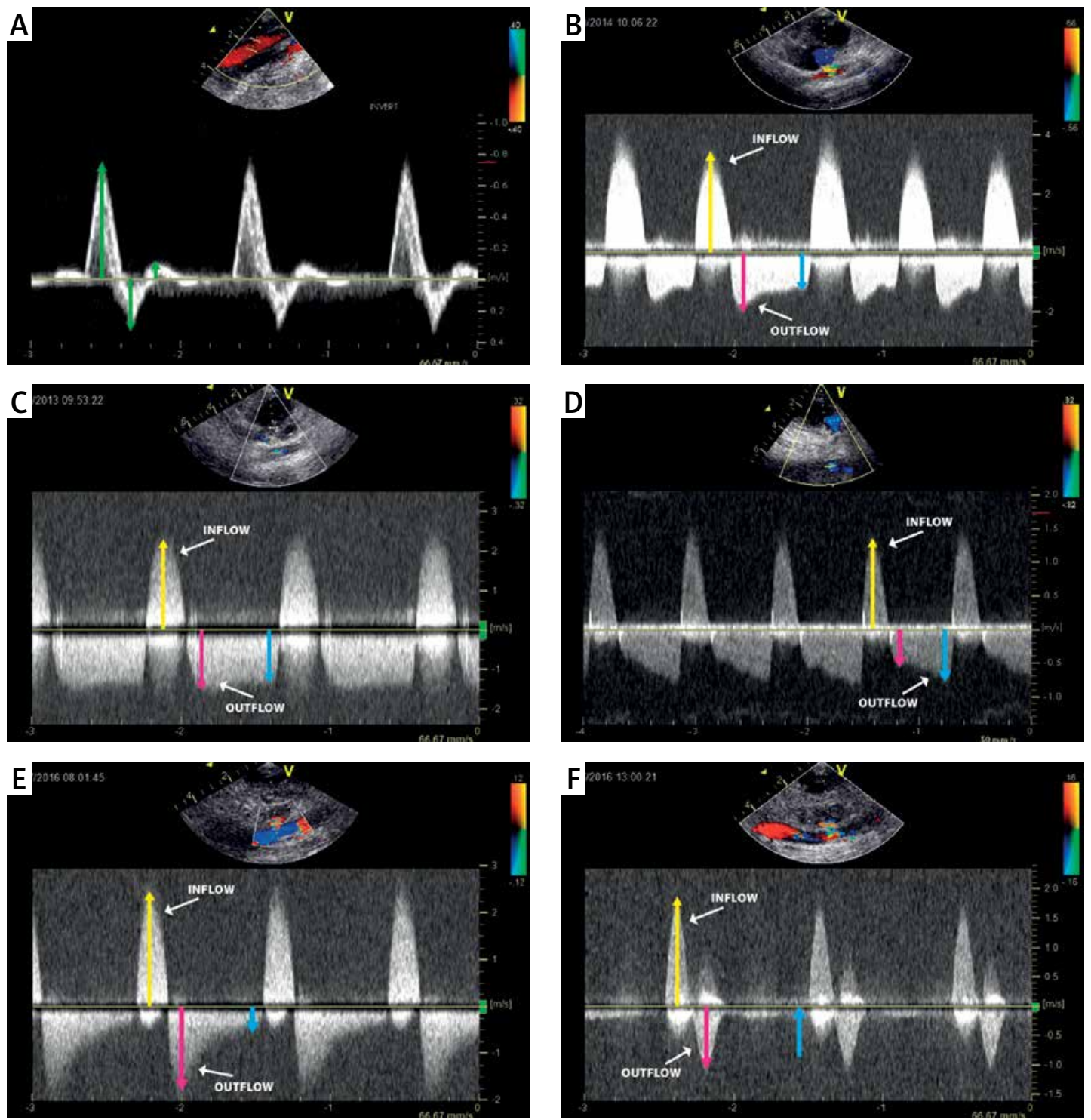

Figure 2. Pulsed wave Doppler ultrasound of the inguinal area with femoral artery pseudoaneurysm. A - Normal three-phasic flow (green arrows) in the common femoral artery. B - Inflow toward (yellow arrow) and early (red arrow) and late (blue arrow) outflow velocities from the pseudoaneurysm. C - Three-phasic flow during embolization. D - Flow in a large pseudoaneurysm with a large mural thrombus. E - Flow in a pseudoaneurysm with a high risk of recanalization. $\mathbf{F}$ - Flow in a pseudoaneurysm diagnosed more than 7 days after vessel cannulation. See additional details in the main text 
groups. The UGTI = 1 group consisted of patients with only one UGTI attempt and no evidence of a psA recurrence. The UGTI > 1 group consisted of patients with a psA recurrence and in which the UGTI treatment was repeated until complete psA obliteration was achieved. There were no specific exclusion criteria. Written informed consent for UGTI was obtained from all patients prior to the treatment. The study was approved by the Bioethics Committee of the local chamber of physicians.

\section{Thrombin injection under the guidance of ultrasound}

The procedure was performed using bovine thrombin ("Thrombin 400" and "BioThrombin 400", Biomed, Wytwórnia Surowic i Szczepionek, Lublin, Poland). It is produced as a sterile powder (one $2 \mathrm{ml}$ vial contains $400 \mathrm{IU}$ ) and a solvent (another $2-\mathrm{ml}$ vial with $0.9 \% \mathrm{NaCl}$ solution). A $1 \mathrm{ml}$ syringe and needles of either $21 \mathrm{G}(0.8 \times 40 \mathrm{~mm})$ or $22 \mathrm{G}$ $(90 \mathrm{~mm})$ were used in the procedure. After the treatment, UGTI patients were kept on bed rest for 16-25 h, with follow-up ultrasonography at 24-72 h, and at 7 days following the procedure. In patients with psA recurrence a subsequent UGTI was performed. The procedure was repeated until complete psA obliteration.

\section{Statistical analysis}

The distribution of the continuous variables was analyzed for normality using the Shapiro-Wilk test. As most of these variables had non-normal distribution, data are shown as median, lower and upper quartiles (Q1-Q3), and minimum and maximum values. The groups were compared using the non-parametric Mann-Whitney $U$ test (unpaired), or the Wilcoxon signed rank test (paired), or Kruskal-Wallis test (more than two unpaired groups) with post-hoc analysis. Categorical variables are presented as percentages and numbers. The groups were compared for two categorical variables using the chi-square test or Fisher's exact test when it was required. Receiver operating characteristic (ROC) analysis was used to obtain a good predictor for UGTI. The area under the curve (AUC), with odds ratio (OR) and 95\% confidence intervals $(\mathrm{Cl})$ and a ROC curve plot, were presented. A $p<0.05$ for two tailed tests was considered significant. All calculations were performed using SPSS Statistics v. 23 and $R$ (version 3.1.2, IBM, USA).

\section{Results}

\section{Patients}

There were no differences in age, sex, body mass index, body surface area, arterial hypertension, and presence of diabetes or dyslipidemia be- tween the groups. Of note, heart failure was significantly more frequent in the UGTI $>1$ group. In $486(95.7 \%)$ patients the common femoral artery or its branches (common 64\%, superficial 32\%, deep 4\%) was identified as the cannulation site, while it was found that in $22(4.3 \%)$ patients the femoral vein (due to inadvertent catheter placement) was the cannulation site (Table II).

\section{Thrombin injection and complications}

Complete psA embolization after the initial procedure occurred in 432 (85\%) patients. In 76 (15\%) patients, a single repeat thrombin injection was necessary for complete treatment. However, 2 injections in 49 patients (64\%), 3 in 15 patients (20\%), and 4 or more in 12 patients (16\%) were required for total ablation. During the first UGTI attempt a thrombin dose $<200$ IU was sufficient in both subgroups (UGTI = 1, UGTI > 1), whereas doses $>600$ IU were most frequent in subsequent treatments in the UGTI > 1 group. The total thrombin dose was also lower in the UGTI = 1 group. In the UGTI > 1 group the first procedure required larger doses of thrombin when compared with the single-procedure group UGTI = 1 , and further attempts in UGTI > 1 were also associated with higher thrombin doses as compared to the first procedure in this group (Figure $3 \mathrm{~A}$ ). During UGTI in $71(14 \%)$ patients, there was an unintentional thrombin leakage from the psA to the vascular lumen resulting in transient paresthesia, numbness, tingling or mild pain. This complication was more common in the UGTI > 1 group. There was one pulmonary embolism in the UGTI = 1 group. In this case, the psA coexisted with an arterio-venous fistula, resulting in the thrombin leakage out of the psA to the femoral vein (Table II). The antiplatelet and anticoagulant medications, in different combinations, were similar in both groups (Table III), and were not associated with the number of UGTI attempts in the UGTI > 1 group.

\section{The recurrent pseudoaneurysm characteristics}

The psA recurrence was more common in patients with a psA volume exceeding $5 \mathrm{~mL}$ and a short communicating channel. A LEVI of $<0.2$ discriminated between the UGTI = 1 and UGTI $>1$ groups, and was observed more frequently in patients with a time interval from the vessel puncture to UGTI exceeding 7 days. In addition, LEVI (AUC $=0.997,95 \% \mathrm{Cl}: 0.952-1 ; 95 \% \mathrm{Cl}$ : 0-0.84) and the time interval between vessel puncture and UGTI were the best predictors for psA recurrence (Figures $3 \mathrm{~B}, \mathrm{D}$ ). The psA recurrence was more common if the time from vessel cannulation to UGTI exceeded 7 days (Figure $3 \mathrm{D}$ ). 
Table II. Baseline characteristics and procedural aspects

\begin{tabular}{|c|c|c|c|}
\hline Parameter & UGTI = $1(n=432)$ & UGTI $>1(n=76)$ & $P$-value \\
\hline Age [years] & $68.0(60-76)$ & $68.0(61-76)$ & 0.962 \\
\hline Female sex & $221(51.2)$ & $41(53.9)$ & 0.709 \\
\hline Arterial hypertension & $329(76.1)$ & $63(82.9)$ & 0.25 \\
\hline Diabetes & $87(20.1)$ & $20(26.3)$ & 0.29 \\
\hline Heart failure & $113(26.1)$ & $70(92.1)$ & $<0.0001$ \\
\hline Dyslipidemia & $260(60.2)$ & $50(65.8)$ & 0.43 \\
\hline Body surface area $\left[\mathrm{m}^{2}\right]$ & $1.81(1.69-1.92)$ & $1.79(1.69-1.94)$ & 0.78 \\
\hline Body mass index $\left[\mathrm{kg} / \mathrm{m}^{2}\right]$ & $23.2(20.7-25.4)$ & $23.1(20.5-24.2)$ & 0.21 \\
\hline Punctured vessel: & & & 0.537 \\
\hline Femoral artery & $415(96.1)$ & $72(94.7)$ & \\
\hline Femoral vein & $17(3.9)$ & $4(5.3)$ & \\
\hline Sheath size [Fr]: & & & 0.606 \\
\hline 6 & $276(63.9)$ & $46(60.5)$ & \\
\hline$>6$ & $156(36.1)$ & $30(40.5)$ & \\
\hline Number of drugs*: & & & 0.300 \\
\hline 0 & $18(4.2)$ & $2(2.6)$ & \\
\hline 1 & $77(17.8)$ & $9(11.8)$ & \\
\hline 2 & $244(56.5)$ & $42(55.3)$ & \\
\hline 3 & $93(21.5)$ & $23(30.3)$ & \\
\hline Time from puncture to UGTI [days]: & & & $<0.001$ \\
\hline$<7$ & $411(95.1)$ & $61(80.3)$ & \\
\hline$\geq 7$ & $21(4.9)$ & $15(19.7)$ & \\
\hline Number of compartments: & & & 0.080 \\
\hline 1 & $306(70.8)$ & $46(60.5)$ & \\
\hline$>1$ & $126(29.2)$ & $30(39.5)$ & \\
\hline Volume of psA [ml] & $2.23(0.81-5.02)$ & $3.09(1.11-8.80)$ & 0.051 \\
\hline Ranges of psA volume [ml]: & & & 0.032 \\
\hline$<1$ & $126(29.2)$ & $17(22.4)$ & \\
\hline $1-5$ & $198(45.8)$ & $29(38.2)$ & \\
\hline$>5$ & $108(25)$ & $30(39.4)$ & \\
\hline Channel length [mm] & $5(0-12.7)$ & $2(0-9.5)$ & 0.037 \\
\hline LEVI & $0.59(0.39-0.73)$ & $0.12(0.02-0.17)$ & $<0.001$ \\
\hline Thrombin dose ${ }^{* *}[\mathrm{IU}]$ & $150(80-220)$ & $200(80-400)$ & 0.025 \\
\hline Ranges of thrombin dose ${ }^{* *}[\mathrm{IU}]$ : & & & 0.037 \\
\hline$<200$ & $259(60)$ & $37(48.7)$ & \\
\hline $200-600$ & $152(35.2)$ & $30(39.5)$ & \\
\hline$>600$ & $21(4.9)$ & $9(11.8)$ & \\
\hline Thrombin dose per patient [IU] & $150(80-220)$ & $520(265-845)$ & $<0.001$ \\
\hline Ranges of thrombin dose per patient [IU]: & & & $<0.001$ \\
\hline$<200$ & $259(60)$ & $13(17.1)$ & \\
\hline $200-600$ & $152(35.2)$ & $31(40.8)$ & \\
\hline$>600$ & $21(4.9)$ & $32(42.1)$ & \\
\hline Complications: & & & 0.001 \\
\hline None & $380(87.2)$ & $56(73.7)$ & \\
\hline Paresthesia & $52(12.0)$ & $19(25.0)$ & \\
\hline Pulmonary embolism & $1(0.2)$ & $0(0)$ & \\
\hline
\end{tabular}

Data are presented as number and percentage or median and interquartile range. UGTI - ultrasound-guided thrombin injection, pSA - pseudoaneurysm, LEVI - late to early velocity index, IU - international unit. ${ }^{\star} E i t h e r$ aspirin or clopidogrel, or heparin, or vitamin $K$ antagonist. ${ }^{* *}$ Per one UGTI. 
A

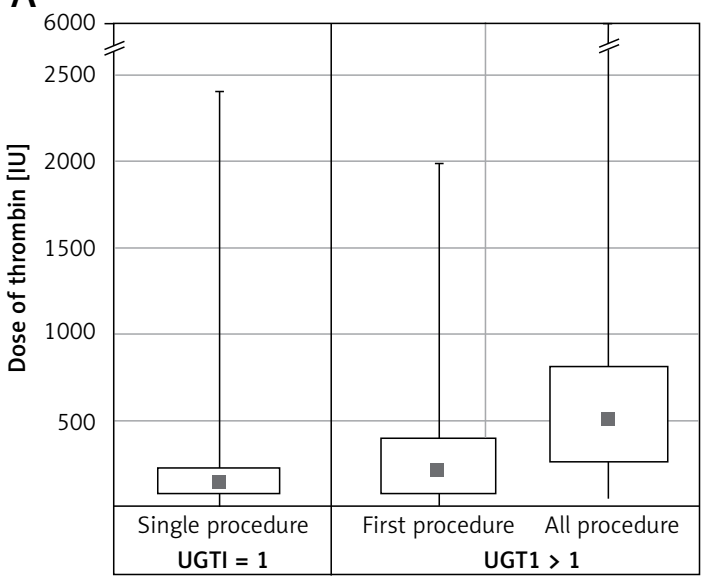

C

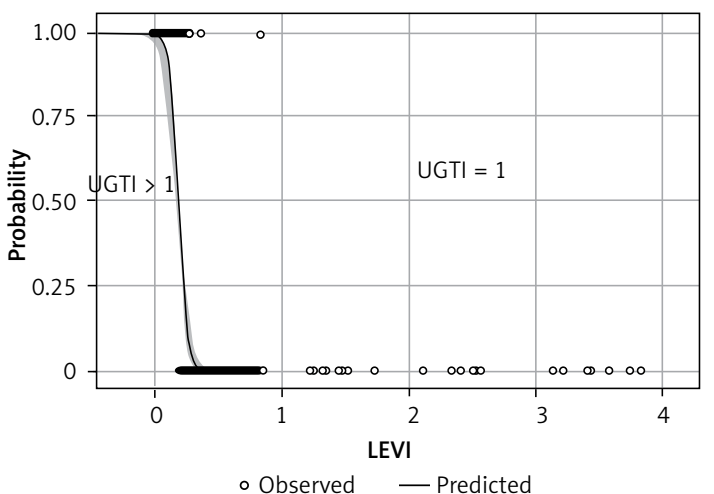

B

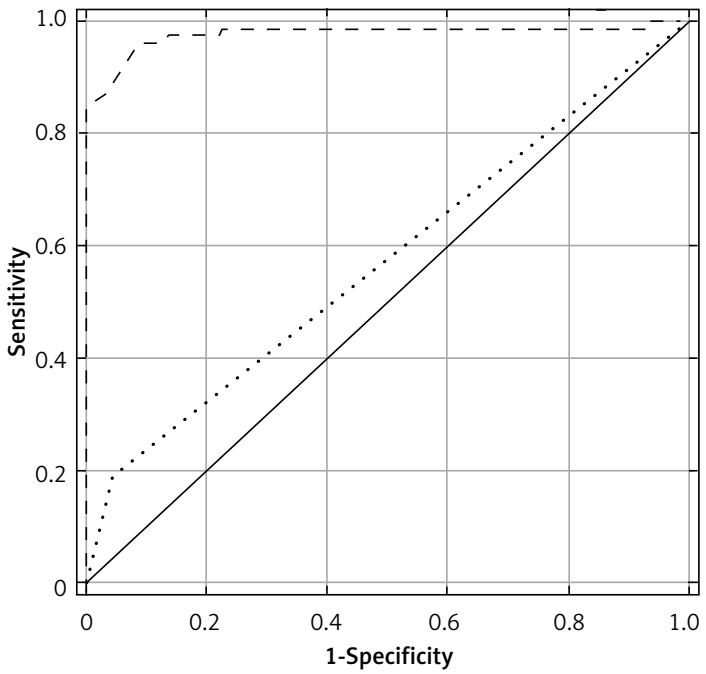

D

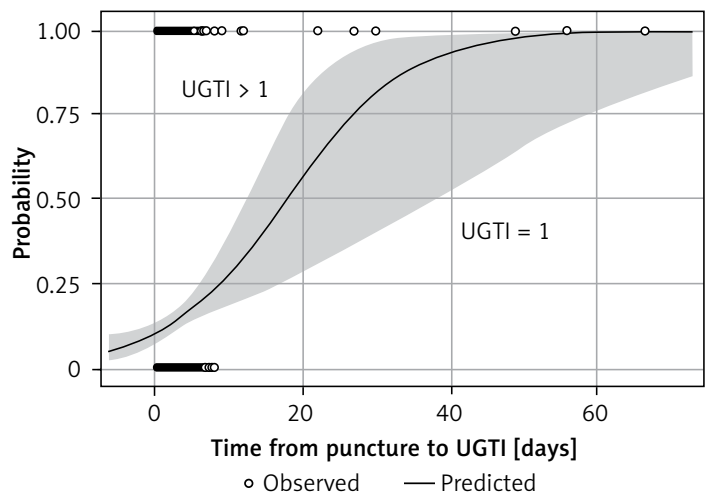

Figure 3. Thrombin doses during single and multiple injections (A). Receiver operating characteristic (ROC) curve for late to early velocity index (LEVI) (dashed line) and time from puncture to thrombin injection (UGTI) $>7$ days (dotted line) (B). Logistic regression fit plot and the ROC curve for LEVI (C) and the time from puncture to UGTI (D)

\section{Discussion}

Seven large studies have reported on the results of UGTI treatment, each of them documenting therapy outcomes in over 100 patients with psA [1, 3, 4, 9-12]. The rate of recanalization has been estimated at 5 to $13 \%$; however, the potential causes have not been investigated or discussed sufficiently (Table I). In our study, after performing more than 500 UGTI treatments, complications were detected in a larger proportion of the total patients (15.6\%). The primary finding of our study is the identification of parameters which predict which patients are at risk for recurrent psA after UGTI. This information is of great clinical importance, as thrombin is a very potent agent with a series of dangerous complications, especially when used in repeated UGTI treatments.

\section{Concomitant medication}

Antiplatelet and anticoagulant therapy remains a gold standard in many transcatheter cardiovas- cular procedures. In the present study, half of the patients received dual antiplatelet therapies (i.e. aspirin + clopidogrel) and $23 \%$ received a combination of three antiplatelet or anticoagulant drugs (i.e. aspirin, clopidogrel or ticlopidine, unfractionated heparin, low molecular weight heparin, vitamin $\mathrm{K}$ antagonist). Other studies have not documented any significant effect of these medications on UGTI treatment success $[4,5,10,11]$. Our findings show that these drugs were not predictive of psA recurrence (Table III). Unfortunately, none of the previous investigations evaluated UGTI success in patients receiving a non-vitamin $\mathrm{K}$ antagonist oral anticoagulant (NOAC). Although there are currently no reports available, it appears reasonable to switch from dabigatran to a different NOAC prior to UGTI, as dabigatran acts as a thrombin inhibitor and may attenuate the effect of UGTI.

\section{Morphology of pseudoaneurysm}

There are several structural types of pseudoaneurysms. Most frequently, they are divided into 
Table III. Concomitant antiplatelet and anticoagulant medication

\begin{tabular}{|c|c|c|c|}
\hline Agent or combination & UGTI = $1(n=432)$ & UGTI $>1(n=76)$ & $P$-value \\
\hline None & $18(4.2)$ & $2(2.6)$ & 0.567 \\
\hline \multicolumn{4}{|l|}{ Single therapy: } \\
\hline Aspirin & $62(14.4)$ & $4(5.3)$ & 0.030 \\
\hline LMWH & $9(2.1)$ & $1(1.3)$ & NA \\
\hline VKA & $5(1.2)$ & $3(3.9)$ & NA \\
\hline UFH & $0(0)$ & $1(1.3)$ & NA \\
\hline Ticlopidine & $1(0.2)$ & $0(0)$ & NA \\
\hline \multicolumn{4}{|l|}{ Double therapy: } \\
\hline Aspirin + clopidogrel & $215(49.8)$ & $36(47.4)$ & 0.711 \\
\hline Aspirin + ticlopidine & $16(3.7)$ & $1(1.3)$ & 0.489 \\
\hline Aspirin + LMWH & $10(2.3)$ & $2(2.6)$ & 0.697 \\
\hline Aspirin + UFH & $2(0.5)$ & $1(1.3)$ & NA \\
\hline VKA + LMWH & $0(0)$ & $2(2.6)$ & NA \\
\hline Ticlopidine + UFH & $1(0.2)$ & $0(0)$ & NA \\
\hline \multicolumn{4}{|l|}{ Triple therapy: } \\
\hline Aspirin + clopidogrel + LMWH & $33(7.6)$ & $6(7.9)$ & 1 \\
\hline Aspirin + clopidogrel + UFH & $54(12.5)$ & $13(17.1)$ & 0.531 \\
\hline Aspirin + ticlopidine + UFH & $4(0.9)$ & $2(2.6)$ & NA \\
\hline Aspirin + clopidogrel + VKA & $2(0.5)$ & $2(2.6)$ & NA \\
\hline
\end{tabular}

Data are presented as number and percentage. $L M W H$ - low molecular weight heparin, UFH - unfractionated heparin, VKA - vitamin $K$ antagonist, NA - not applicable.

unilobed and multilobed, with a communicating channel or an opening between the psA cavity and the affected artery [3, 4, 9-11]. Unfortunately, the psA morphology has not been discussed in detail in patients with recanalization. Our study shows that the failure of primary UGTI treatment is likely related to a psA volume exceeding $5 \mathrm{ml}$ $(p=0.032)$. It is possible that primary injection of thrombin into a distal psA cavity (recommended in multilobed psA) prevents an adequate amount of thrombin from entering the proximal cavity. As such, the thrombus formations may be less stable than in the distal cavity. Conversely, in a psA with a large single cavity, thrombin is generally injected as far as possible away from the neck, resulting in this region having low clot stability as well.

\section{Time interval from pseudoaneurysm formation to thrombin injection}

When UGTI is performed a substantial time period after the initial artery puncture, there is often a need for repeat or multiple UGTI treatments $[4,11]$. In our study, a delay in UGTI initi- ation greater than 7 days was a significant risk factor for psA recurrence. While direct evidence remains elusive, we hypothesize that a long delay in the diagnosis of a psA may cause morphological remodeling of the inner lining of the psA cavity and false channel. As a result, this could have a negative effect on clot stabilization. To the best of our knowledge, no previous study has reported data on the consequences of the time period between the vessel puncture and UGTI. As such, our study is the first to suggest this potentially important finding, which could impact future patient care.

\section{Thrombin dose}

In the largest studies previously conducted, the average combined thrombin dose during UGTI ranged from 200 to $450 \mathrm{IU}$ [1, 3, 4, 9-11]. Despite different modalities of thrombin application, an average single bolus of $100 \mathrm{IU}$ of thrombin at a time was injected in these previous studies. In our study we achieved a UGTI success rate of $97 \%$ with a lower average total thrombin dose of $150 \mathrm{IU}$. In patients with psA recurrence, significantly more 
thrombin was required in the second UGTI treatment. Furthermore, the amount of thrombin used during the first procedure in the UGTI > 1 group was significantly higher when compared to the single procedure in the UGTI = 1 group. This could potentially increase the risk of thrombin leak towards the arterial lumen. Other investigators have not provided sufficient data on the thrombin dose used in the repeated UGTI, and as such our findings are the first to present these data.

\section{Spectral Doppler waveform analysis}

Following a precise analysis of the spectral Doppler waveform, we hypothesize that early and late velocities are not arranged randomly. The relationship between these velocities has not been studied previously. Therefore, we proposed the LEVI as a novel parameter for psA recurrence risk assessment. We observed that LEVI depended on the psA cavity size, or the stage of clot formation and retraction. A normal arterial Doppler spectrum is three-phasic (Figure $2 \mathrm{~A}$ ). It appears that most frequently the early outflow velocity is greater than the late velocity, and therefore LEVI is less than 1.0 (Figure 2 B). In the case of psA, "the wall" seems to be a simplification, as the newly developed psA has no true, unique wall; it is limited by the surrounding tissues as well as a flaccid fresh thrombus adhering to the peripheral borders. We observed that this is particularly valid if the psA is diagnosed early (e.g. less than 7 day after the index procedure). A rigid wall is often present in an older psA (existing far longer than 7 days). The high early outflow velocity is a result of the compliant nature of the parietal thrombus, as well as the soft tissues surrounding the psA. Elasticity propels the largest amount of blood at the beginning of outflow, then the flow decreases until the beginning of the next inflow. In contrast, during UGTI, when the psA cavity is partially filled with a fresh thrombus, its compression at the early beginning of outflow results in a flat spectral Doppler waveform and LEVI equals 1.0 (Figure $2 \mathrm{C}$ ). When the psA cavity is large, LEVI may be even greater than 1.0. We hypothesize that the parietal thrombus might be responsible for this specific pattern, as at the beginning of outflow the thrombus compression absorbs energy, and the velocity is lower than at the end of this phase (Figures 1 B, 2 D). In our study, patients with the psA recurrence after UGTI had a flow velocity that was very low in the late outflow phase when compared to the initial value. A LEVI of less than 0.2 was a significant predictor of a psA recurrence. In subjects with a late diagnosis of psA, the spectral Doppler waveform seems to be atypical, as it is very short and steep and may mimic arterial blood flow, but at higher velocities and without the third phase (Figure $2 \mathrm{~F}$ ).
In such patients LEVI equals 0 , because no blood flow is recorded at the end of outflow, prior to the next cycle.

\section{latrogenic immunization after UGTI}

Administration of a heterologous substance, such as bovine thrombin, during UGTI may cause iatrogenic immunization. Exposure to thrombin during a second procedure may potentiate these immune responses [21]. In our study, none of the patients, despite multiple UGTI attempts, developed an immunization reaction. This was most likely due to the application of thrombin in short intervals. Indeed, more than four UGTI attempts were made within 5 days in some cases. It was possible this occurred because the patients had not had exposure to bovine thrombin previously. Some investigators have previously demonstrated that such reactions can range from mild clinical symptoms, such as fever, hypotension, bradycardia or urticaria, to full anaphylaxis [3, 22, 23]. Multiple attempts at UGTI treatment have already been reported, but without specifying the safe time interval for repeat thrombin injection [4, 9, 10]. Although no specific data are available, we hypothesize that it would be reasonable to choose human thrombin in patients previously exposed to a bovine treatment.

\section{Complications of UGTI}

Potential complications of thrombin injection were clearly described in a review paper by Webber et al. [17], and in a small sample of 50 subjects by Krueger et al. [5]. Evidence from large studies indicates that UGTI rarely causes serious complications, such as arterial or pulmonary embolism [3, 4, 10-12, 17, 18, 24, 25]. However, mild complications can result from unintentional thrombin leak from the psA cavity towards the native circulation, as a delay of 3 to $90 \mathrm{~s}$ (15 s on average) between thrombin injection and thrombus formation has been reported $[4,9,11]$. When this occurs, transient paresthesia, numbness, tingling or mild pain can be observed; however, they resolve within minutes due to native fibrinolytic activity. Paresthesia during UGTI was previously detected in several studies, but no in-depth analysis of the event has been performed [4, 9]. However, it has previously been suggested to occur in up to $15 \%$ of patients undergoing UGTI [11]. In our analysis, paresthesia developed in $14.1 \%$ of patients, including those at increased risk (i.e. those in need of repeated UGTI). Other complications included venous thrombosis, hematoma, and infection. However, it seems they were related to the psA structure or too late diagnosis, rather than the procedure itself $[3,4,17,10]$. 


\section{A new technique for the treatment of pseudoaneurysm}

Recently, a new option for the treatment of psA has been proposed [26]. Ultrasound-guided tissue glue injection is a similar technique to UGTI. Its main advantage is simultaneous application of thrombin and fibrinogen, which rapidly generates a stabilized thrombus before the first outflow. As it is a costly method, it should be reserved primarily for patients requiring multiple attempts.

\section{Study limitations}

Although we were able to prospectively include a large number of patients, our study is lacking a separate low and high LEVI comparison, and prognostic significance of this measurement. However, the rate of psA recurrence is relatively low, and the number of patients necessary to ensure statistical power could be too small, or the inclusion period could be too long, to obtain reliable results at this time.

In conclusion, the rate of psA recurrence after UGTI was approximately $15 \%$. We propose that the LEVI in spectral Doppler waveform analysis is a novel parameter to select psA patients who may be prone to recurrence. A LEVI of less than 0.2 , a time interval between the vessel cannulation and a psA diagnosis longer than 7 days, a psA volume exceeding $5 \mathrm{~mL}$, and a short communicating channel between the psA cavity and the artery, all predicted psA recurrence after UGTI. However, we found that adjunctive pharmacotherapy (i.e. antiplatelet and anticoagulants in different combinations) did not increase the recurrence risk.

\section{Acknowledgments}

The project is supported under the program of the Minister of Science and Higher Education under the name "Regional Initiative of Excellence" in 2019-2022 project number: 024/RID/2018/19, financing amount: 11.999.000,00 PLN.

\section{Conflict of interest}

The authors declare no conflict of interest.

\section{References}

1. Maleux G, Hendrickx S, Vaninbroukx J, et al. Precutaneous injection of human thrombin to treat iatrogenic femoral pseudoaneurysm: short and midterm ultrtasound follow-up. Eur Radiol 2003; 13: 209-12.

2. Ahmed F, Turner S, Torrie P, et al. latrogenic femoral artery pseudoaneurysms - a review of current methods of diagnosis and treatment. Clin Radiol 2008; 63: 1310-6.

3. Krueger K, Zaehringer M, Strohe D, et al. Postcatheterization pseudoaneurysm: results of US-guided percutaneous thrombin injection in 240 patients. Radiology 2005; 236: 1104-10.
4. Hofmann I, Wunderlich N, Robertson G, et al. Percutaneous injection of thrombin for the treatment of pseudoaneurysm: the German multicentre registry. Eurolntervention 2007; 3: 321-6.

5. Kruger K, Zahringer M, Sohngen FD, et al. Femoral pseudoaneurysms: management with precutaneous thrombin injections success rate and effects on systemic coagulation. Radiology 2003; 226: 452-8.

6. Calton WC, Franklin DP, Elmore JR, et al. Ultrasound-guided thrombin injection is a safe and durable treatment for femoral pseudoaneurysms. Vasc Surg 2001; 35: 379-83.

7. La Perna L, Olin J, Goines D, et al. Ultrasound-guided thrombin injection for the treatment of postcatheterization pseudoaneurysms. Circulation 2000; 102: 2391-5.

8. Vazquez V, Reus M, Pinero A, et al. Human thrombin for treatment of pseudoaneurysms: comparison of bovine and human thrombin sonogram-guided injection. AJR Am J Roentgenol 2005; 184: 1665-71.

9. Paulson EK, Nelson RC, Mayes CE, et al. Sonographically guided thrombin injection of iatrogenic femoral pseudoaneurysms: further experience of a single institution AJR Am J Roentgenol 2001; 177: 309-16.

10. Schneider C, Malisius R, Kuchler R, et al. A prospective study on ultrasound-guided percutaneous thrombin injection for treatment of iatrogenic post-catheterisation femoral pseudoaneurysms. Int J Cardiol 2009; 131:356-61.

11. Kurzawski J, Sadowski M, Janion-Sadowska A. Complications of percutaneous thrombin injection in patients with postcatheterization femoral pseudoaneurysm. J Clin Ultrasound 2016; 44: 188-95.

12. Khoury M, Rebecca A, Greene K, et al. Duplex scanning-guided thrombin injection for the treatment of iatrogenic pseudoaneurysms. J Vasc Surg 2002; 35: 517-21.

13. Snack T, Bilgic, S Ustuner E. Endovascular stent-graft treatment of a traumatic vertebral artery pseudoaneurysm and vertebrojugular fistula. Korean J Radiol 2008; 9: S68-S72.

14. Thalhammer C, Kirchherr S, Uhlich F, et al. Postcatheterization pseudoaneurysms and arteriovenous fistulas. Repair with percutaneous implantation of endovascular covered stents. Radiology 2000; 214: 127-31.

15. Morgan R, Belli AM. Current treatment methods for post-catheterization pseudoaneurysms. Vasc Interv Radiology 2003; 14: 697-710.

16. Garcia E, Gonzalez-Fajardo JA, Gutierrez V, et al. Femoral pseudoaneurysms post-cardiac catheterization surgically treated: evolution and prognosis. Interact Cardio Vasc Thorac Surg 2009; 8: 353-7.

17. Webber GW, Jang J, Gustavson S, et al. Contemporary management of postcatheterization pseudoaneurysms. Circulation 2007; 115: 2666-74.

18. Hamraoui K, Ernst S, van Dessel P. Efficiency and safety of percutaneous treatment of iatrogenic femoral artery pseudoaneurysm by biodegradable collagen injection. J Am Coll Cardiol 2002; 39:1297-304.

19. Onal B, Ilgit ET, Akpek S, et al. Postcatheterization femoral arteriovenous fistula: endovascular treatment with N-butyl-cyanoacrylate embolization. Cardiovasc Intervent Radiol 2006; 29: 276-8.

20. Matson M, Morgan R, Belli A. Precutaneous treatment of pseudoaneurysm using fibrin adhesive. $\mathrm{Br} J$ Radiol 2001; 74: 690-4.

21. Caroll JF, Moskovitz KA, Edward NM. Immunological assessment of patients treated with bovine fibrinogen as a hemostatic agent. Thromb Haemost 1996; 76: 925-31. 
22. Ortel TL, Charles LA, Keller FG, et al. Topical thrombin and acquired coagulation factor inhibitors: clinical spectrum and laboratory diagnosis. Am J Hematol 1994; 45: 128-35.

23. Pope M, Johnston KW. Anaphylaxis after thrombin injection of a femoral pseudoaneurysm: recommendations for prevention. J Vasc Surg 2000; 32: 190-1.

24. Villanueva-Benito I, Solla-Ruiz I, Rodriguez-Calveiro R, Maciñeiras-Montero IL, Rodriguez-Paz CM, Ortiz-Saez A. latrogenic subclavian artery pseudoaneurysm complicating a transradial percutaneous coronary intervention. JACC Cardiovasc Interv 2012; 5: 360-1.

25. Walker TG, Geller SC, Brewster DC. Transcatheter occlusion of a profunda femoral artery pseudoaneurysm using thrombin. Am J Roentgenol 1987; 149: 185-6.

26. Kurzawski J, Janion-Sadowska A, Sadowski M. A novel minimally invasive method of successful tissue glue injection in patients with iatrogenic pseudoaneurysm. Br J Radiol 2018; 91: 20170538. 